\title{
Blood Pressure Indices and Associated Risk Factors in a Rural West African Adult Population: Insights from an AWI-Gen Substudy in Ghana
}

\author{
Godfred Agongo ${ }^{(D)},{ }^{1,2,3}$ Engelbert A. Nonterah, ${ }^{1,4}$ Lucas Amenga-Etego, ${ }^{5}$ \\ Cornelius Debpuur, ${ }^{1}$ Michael B. Kaburise, ${ }^{1}$ Stuart A. Ali, ${ }^{2}$ Nigel J. Crowther, \\ Michèle Ramsay, ${ }^{2,3}$ and Abraham R. Oduro' \\ ${ }^{1}$ Navrongo Health Research Centre, Navrongo, Ghana \\ ${ }^{2}$ Sydney Brenner Institute for Molecular Bioscience, Faculty of Health Sciences, University of the Witwatersrand, \\ Johannesburg, South Africa \\ ${ }^{3}$ Division of Human Genetics, National Health Laboratory Service and School of Pathology, Faculty of Health Sciences, \\ University of the Witwatersrand, Johannesburg, South Africa \\ ${ }^{4}$ Julius Global Health, Julius Center for Health Sciences and Primary Care, University Medical Centre Utrecht, Utrecht University, \\ Utrecht, Netherlands \\ ${ }^{5}$ West African Centre for Cell Biology of Infectious Pathogens, Department of Biochemistry, Cell and Molecular Biology, \\ University of Ghana, Accra, Ghana \\ ${ }^{6}$ Department of Chemical Pathology, National Health Laboratory Service and School of Pathology, Faculty of Health Sciences, \\ University of the Witwatersrand, Johannesburg, South Africa
}

Correspondence should be addressed to Godfred Agongo; godfred.agongo@navrongo-hrc.org

Received 9 June 2019; Accepted 17 March 2020; Published 27 April 2020

Academic Editor: Kwok Leung Ong

Copyright (c) 2020 Godfred Agongo et al. This is an open access article distributed under the Creative Commons Attribution License, which permits unrestricted use, distribution, and reproduction in any medium, provided the original work is properly cited.

Systolic (SBP) and diastolic blood pressure (DBP) are commonly used for cardiovascular disease (CVD) risk prediction, and pulse pressure (PP) and mean arterial blood pressure (MAP) can provide additional information. It is therefore important to understand the factors associated with these cardiovascular risk markers. This cross-sectional study involved 1839 men and women aged 40-60 years. Data on SBP, DBP, MAP, PP, sociodemography, lifestyle, anthropometry, and lipids were collected. Genderstratified linear regression analyses were performed to determine the association between log-transformed blood pressure indices and the study variables. Age was associated with all measured blood pressure indices $(p<0.001)$ among men and women. Men had higher SBP $(p=0.007)$ and PP $(p<0.001)$ than women. Nankana ethnicity was associated with higher PP levels $(p<0.005)$ in the total population. Vendor meal consumption among women was associated with higher PP levels $(p<0.05)$. Fruit intake among men was associated with lower PP levels $(p<0.05)$. Currently unmarried women had higher SBP $(p<0.005), \mathrm{DBP}(p<0.05)$, MAP $(p<0.005)$, and PP $(p<0.005)$ than currently married women. Pesticide exposure was negatively associated with SBP $(p<0.005)$, DBP $(p<0.005)$, MAP $(p<0.005)$, and PP $(p<0.05)$ among women. Increased subcutaneous fat was associated with $\mathrm{DBP}(p<0.005)$ and MAP $(p<0.05)$ among women. Among men, hip circumference was associated with higher DBP and MAP $(p<0.05$ for both associations), subcutaneous fat associated with higher SBP $(p<0.005)$, DBP $(p<0.001)$, and MAP $(p<0.001)$ and visceral fat was associated with higher PP $(p<0.05)$. In the total population, visceral fat was associated with higher DBP $(p<0.05)$ and MAP $(p<0.001)$. High-density lipoprotein cholesterol was positively associated with SBP $(p<0.005)$, DBP $(p<0.005)$, and MAP $(p<0.001)$ for women and positively associated with SBP, DBP, and MAP $(p<0.001$ for all three $)$ and PP $(p<0.05)$ for men. The association of blood pressure indices with modifiable risk factors suggests that targeted health interventions may reduce CVD risk in this population. 


\section{Introduction}

Though the prevalence of cardiovascular diseases (CVDs) is greater in high-income than low- and middle-income countries (LMIC), the rate of premature CVD-related deaths is higher in LMIC [1]. One of the key CVD risk factors is hypertension, which is estimated to account for nearly 10.4 million deaths worldwide annually and is a major cause of noncommunicable disease-related deaths in sub-Saharan Africa (SSA) [2, 3]. In 2010 the prevalence of hypertension among adults was estimated at 1.39 billion persons globally, representing $31 \%$ of all adults [4]. In both rural and urban Ghanaian settings, the prevalence of hypertension in adults is reported to range from 19 to 55\% [5-7]. This is similar to the estimates in other LMIC, suggesting that Ghana is not spared in the global epidemic [8].

The prevalence of hypertension in the two KassenaNankana districts (KNDs) of rural northern Ghana is high. A study from the late 1990s reported a prevalence of $19.3 \%$ in the KNDs [9]. Findings from the recent H3Africa AWIGen project show an increase in the prevalence of hypertension to $24.8 \%$ in adults aged $40-60$ years [10]. These findings suggest that the prevalence of hypertension in this rural setting is gradually rising and that hypertension in Ghana is not only an urban problem but a major burden in rural communities as well. In addition to the rising prevalence of hypertension, underdiagnosis and poor control of blood pressure in diagnosed hypertensive subjects have been reported $[7,10,11]$. Fully understanding the determinants of the indices of blood pressure will contribute to holistic management of hypertension.

Although hypertension is diagnosed using systolic blood pressure (SBP) and diastolic blood pressure (DBP), CVD risk may equally be predicted by analyzing pulse pressure (PP) (the difference between SBP and DBP) [12-14], and mean arterial pressure (MAP) (the sum of DBP and one-third of pulse pressure $(\mathrm{DBP}+(\mathrm{PP} / 3))$ [14]. Pulse pressure is an important predictor in cardiovascular events and predicts the risk of congestive heart failure in the elderly [15], while MAP is the perfusion pressure that is encountered by end organs including liver and kidney [16]. Therefore, combining these blood pressure indices is superior to a single component in predicting CVD, as these may provide additional and holistic pathophysiological information on hypertension, particularly in adults [17]. In combating hypertension, it is important to determine which modifiable risk factors influence these indices so as to inform nonpharmacological measures for targeting the lowering of blood pressure and prevention of CVD in adult men and women.

Studies globally and in urban Ghanaian settings have attributed rising blood pressure to sociodemographic determinants including age and educational status, environmental factors such as exposure to pesticides and alcohol consumption, unfavourable lipid levels, and particular anthropometric indices [18-23]. The question remains as to whether these factors also influence levels of blood pressure among rural Ghanaian adults. An earlier study that looked at the epidemiology of blood pressure in rural northern Ghana investigated mainly anthropometric variables but did not evaluate the influence of other sociodemographic factors or lipid levels on blood pressure indices [9]. Also, the H3Africa AWI-Gen project, in which this study is nested, reported the prevalence of hypertension but did not investigate the factors associated with blood pressure levels in these communities [10]. Furthermore, studies in other African settings have investigated mainly SBP and DBP [24-26] and the factors associated with them, but there is a paucity of data on the factors associated with MAP and PP [27, 28]. This study is the first to report on a wide array of factors associated with SBP, DPB and their derivatives (PP and MAP) in a black African population and therefore provides new knowledge on the association of blood pressure indices with these markers among black Africans and rural northern Ghanaian adults in particular.

\section{Methods}

2.1. Study Setting and Population. The study was conducted in the two Kassena-Nankana districts of northern Ghana which are covered by the Navrongo Health and Demographic Surveillance System (NHDSS). The districts are divided into five zones: central, east, west, north, and south zones. These zones cover a total land area of $1675 \mathrm{~km}^{2}$ and share a border with Burkina Faso in the northwest. Each of the zones is further divided into clusters, which consist of households (Figure 1). The NHDSS currently monitors 165,000 individuals in 32,000 households. The population density of the districts is $91.5 \mathrm{~km}^{2}$ and the major ethnic groups are the Nankana and Kassena. The male to female ratio of the population is 0.92 , with an estimated population growth of $0.81 \%$ [29].

This study is a part of the H3Africa AWI-Gen project that recruited men and of 40-60 years-of-age [30] mainly in the west and north zones, which is home to the Kassem speaking communities and the east zone which is home to the Nankam speaking communities. Participants of the AWI-Gen project were residents within the study area for at least ten years. Pregnant women and participants who were unable to stand for their height to be measured were excluded from the project.

2.2. Study Design and Sampling. The H3Africa AWI-Gen study was a population-based cross-sectional study with stratified random sampling. First, the east, west, north, and south zones were selected from the five zones of the district. Twenty-five clusters each were further randomly sampled from each selected zone using the NHDSS [29]. The list of all men and women aged 40-60 years were generated from each zone. From this list, a sample size of 2200 including $10 \%$ for nonresponse was randomly generated with a fraction of this number randomly sampled from each cluster in proportion to the population size of that cluster. 


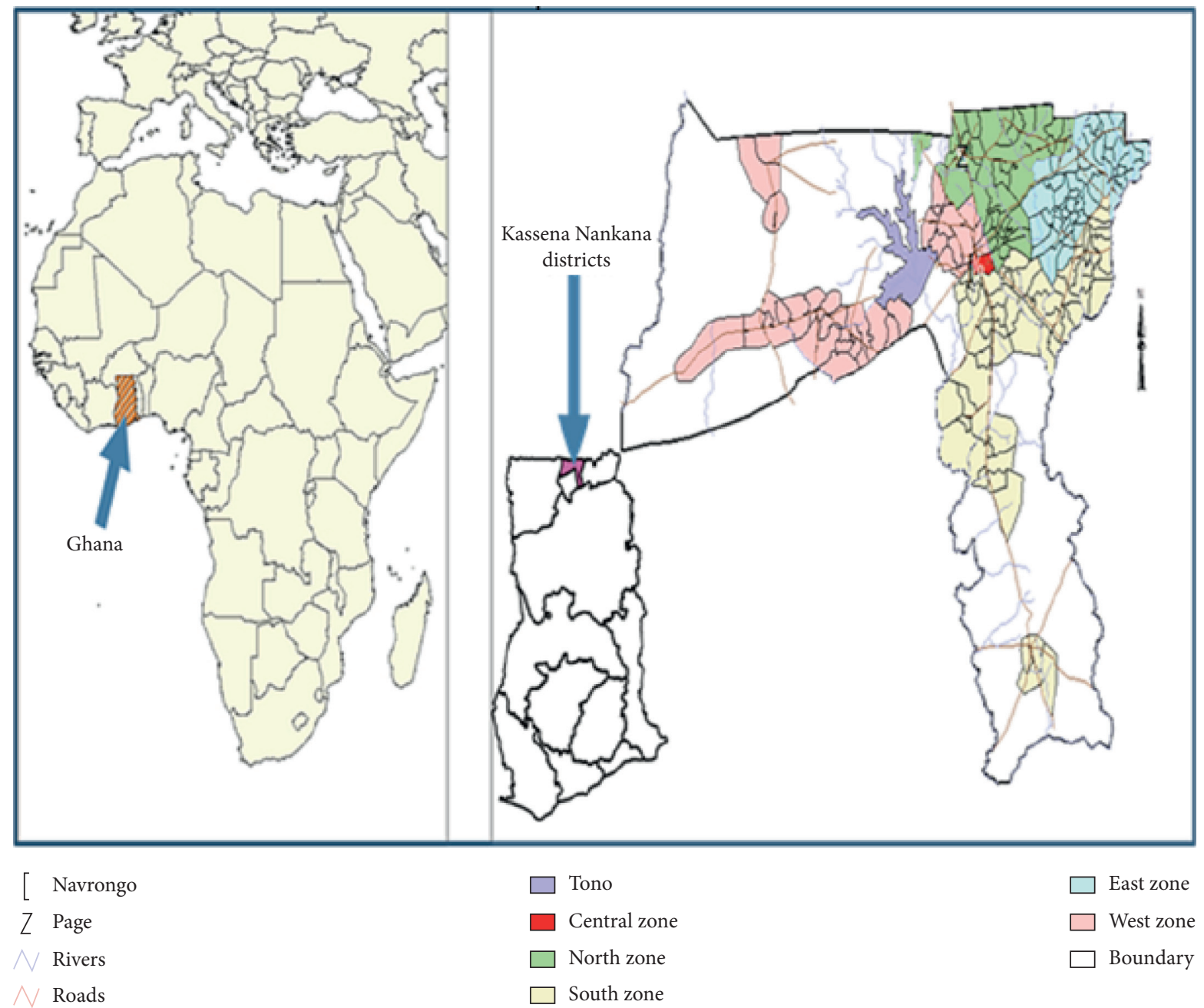

Figure 1: Location of the Kassena-Nankana districts with the zones represented under the Navrongo demographic surveillance system in the upper east region of Ghana.

2.3. Data Collection. Participants were asked to fast the night prior to blood sampling. On the day of data collection information on sociodemographic factors including age, soscioeconomic status (SES), marital status and educational status, and lifestyle factors such as alcohol consumption, tobacco smoking, pesticide exposure, diet, and physical activity (measured as moderated-to-vigorous physical activity (MVPA)) were collected by trained field workers using a structured questionnaire in a language that the participants understood, using methods that have been described elsewhere [31]. Fasting blood was taken by phlebotomists for lipid analyses. High-density lipoprotein cholesterol (HDLC), low-density lipoprotein cholesterol (LDL-C), total cholesterol (TC), and total cholesterol were all measured directly [31, 32]. Three measurements of systolic and diastolic blood pressure were taken using a digital sphygmomanometer (Omron M6, Omron, Kyoto, Japan) and the first readings were discarded while an average of the second and third was taken as the measured blood pressure [32]. Anthropometric measures (weight, height, waist and hip circumference, and visceral and subcutaneous adipose tissue thickness) were obtained as described previously [31, 32].
The data were checked for inconsistencies, missingness, and outliers before it was uploaded into the Research electronic data capture (REDCap) platform [33]. After this, further quality control processes were performed on $10 \%$ of the data.

2.4. Definition of Pesticide Exposure, SBP, and DBP Derivatives. Pesticide exposure was defined by self-reported current working with pesticide or living close to a farm where the pesticide was being used [31]. Pulse pressure (PP) was defined as SBP minus DBP [28], while MAP was defined as the sum of DBP and a third of the PP [17].

2.5. Ethics Approval and Consent to Participate. The study was approved by the Human Research Ethics Committee (HREC) of the University of the Witwatersrand (ID No: M12109), the Ghana Health Service Ethics Review Committee (ID No: GHS-ERC:05/05/2015), and the Navrongo Health Research Centre Institutional Review Board (ID No: NHRCIRB178). Community engagement was carried out in the communities where participants were sampled. Individual informed consent, evidenced by a thump-printed or 
signed informed consent form witnessed by a researcher, was sought from participants before being recruited into the study.

2.6. Statistical Analyses. Analyses were performed using Stata 14.2 (StataCorp, College Station, Texas, 77845, US). Due to nonnormal distribution, blood pressure indices (SBP, DBP, MAP, and PP) were presented as medians and interquartile ranges and compared between men and women using Mann-Whitney $U$ tests and were log-transformed to normality before regression analyses. Gender-stratified multivariable linear regression analyses were performed to determine the association between log-transformed blood pressure indices and each of the study variables. These variables were included in univariate regression analyses against each of the blood pressure indices, and all those that correlated at $p<0.20$ were included in multivariable linear regression models for the appropriate blood pressure variable. Multicollinearity was assessed using the variance inflation factor (VIF) and only variables with a VIF $<5.0$ were included in the models. Association tests at the multivariable regression level were considered statistically significant at $5 \%$ significance level.

\section{Results}

A sample size of 2016 was recruited for this study but study participants who did not have data for one or more of the investigated variables were excluded. Therefore the final sample size was 1839 comprising 993 women and 846 men.

3.1. Basic Characteristics of the Study Population. The general characteristics of the study population are shown in Table 1. The median age of the population was 51 years with that of women being significantly higher $(p<0.001)$. Formal education $(p<0.001)$, employment $(p=0.035)$, marital status $(p<0.001)$, socioeconomic status $(p<0.001)$, current alcohol intake $(p<0.001)$, and current tobacco use $(p<0.001)$ were significantly lower among women than men. However, there was no difference in current smokeless tobacco use between men and women $(p=0.997)$. Vegetable servings per day $(p=0.002)$ and the number of vendor meals per week $(p<0.001)$ were higher among men than women but there was no difference in the number of fruit servings per day between men and women $(p=0.094)$. Women were less physically active and slept longer than men $(p<0.001$ for both). Except for visceral fat, which was higher in men $(p<0.001)$, all the anthropometric indices (BMI, waist circumference, hip circumference, and subcutaneous fat) were higher among women $(p<0.001$ for all). Except for HDL-C, which was significantly lower $(p=0.002)$ and TC, which was significantly higher $(p=0.038)$ in women, there were no significant differences in LDL-C $(p=0.427)$ and TG ( $p=0.770)$ between genders.

The levels of blood pressure indices stratified by gender are shown in Table 2. The median SBP of the study population was 121 (109-135) $\mathrm{mmHg}$ with that of men being significantly higher than that of women $(p=0.007)$.
Similarly, the PP of men was significantly higher than that of women $(p<0.001)$ with a population median PP being 45.0 (38.0-53.0) $\mathrm{mmHg}$. There were, however, no differences in $\operatorname{DBP}(p=0.719)$ and MAP $(p=0.340)$ between men and women.

3.2. Factors Associated with Blood Pressure Indices. The factors associated with blood pressure indices (SBP, DBP, $\mathrm{PP}$, and MAP) were stratified by gender and the results are shown in Tables 3-6. The adjusted $\beta$-coefficients shown in each table are unstandardised and are those derived from multivariable linear regression models that included all the variables that gave $p<0.20$ in the univariate models (Table S1-S3).

Factors associated with SBP are shown in Table 3. Age $(\beta=0.005, p<0.001)$, male gender $(\beta=0.044, p<0.001)$, being currently an unmarried woman $(\beta=0.024, p<0.005)$, subcutaneous fat thickness $(\beta=0.039, p<0.001)$, and HDLC $(\beta=0.054, p<0.001)$ were associated with increased SBP among the total population. Pesticide exposure among women was associated with decreased SBP $(\beta=-0.020$, $p<0.005)$. Together all these factors contributed to $9.5 \%$ of the variance in SBP for the total population.

The factors that were associated with increased DBP among the total population were age $(\beta=0.004 ; p<0.001)$, hip circumference $(\beta=0.020 ; p<0.05)$, visceral fat thickness $(\beta=0.010 ; p<0.05)$, subcutaneous fat thickness $(\beta=0.076$; $p<0.001)$, and HDL-C $(\beta=0.064 ; p<0.001)$. Among women, being currently unmarried $(\beta=0.023$; $p<0.05)$ was associated with increased DBP, while pesticide exposure $(\beta=-0.028 ; p<0.005)$ was associated with decreased DBP. All these factors contributed to a $10.2 \%$ variance in DBP in the total population (Table 4).

Factors associated with MAP in the study population are shown in Table 5 . Age $(\beta=0.004 ; p<0.001)$, visceral fat thickness $(\beta=0.012 ; p<0.001)$, subcutaneous fat thickness $(\beta=0.035 ; p<0.001)$, and HDL-C level $(\beta=0.057 ; p<0.001)$ were associated with increased MAP in the total population while among women pesticide exposure $(\beta=-0.031$; $p<0.005)$ was associated with decreased MAP and being unmarried was associated with a higher MAP $(\beta=0.030$; $p<0.005)$. In men, hip circumference was associated with a higher MAP $(\beta=0.019 ; p<0.05)$. These factors accounted for $9.5 \%$ variance in MAP in the total population.

Age $(\beta=0.009 ; p<0.001)$, male gender $(\beta=0.079$; $p<0.001)$, Nankana ethnicity $(\beta=0.042 ; p<0.005)$, being an unmarried woman $(\beta=0.044 ; p<0.005)$, and vendor meal consumption per month among women $(\beta=0.0004$; $p<0.050)$ were associated with increased PP. Pesticide exposure among women $(\beta=-0.042 ; p<0.050)$ and fruit servings per week among men $(\beta=-0.001 ; p<0.050)$ were associated with decreased PP. These factors explain $5.3 \%$ of the variance in PP in the total population.

\section{Discussion}

The key findings of this study were that all the blood pressure indices increased with increasing age among the total study 
TABLE 1: General characteristics of the rural adult Ghanaian study population stratified by gender and for the combined population.

\begin{tabular}{|c|c|c|c|c|}
\hline Variables & Men $(n=846,46 \%)$ & Women $(n=993,54 \%)$ & Total $(N=1839)$ & $p$ value \\
\hline Age (years) & $50(40-66)$ & $52(40-60)$ & $51(40-60)$ & $<0.001$ \\
\hline \multicolumn{5}{|l|}{ Ethnicity } \\
\hline Kassena & $439(51.9)$ & $516(52.0)$ & $955(51.9)$ & \multirow[t]{3}{*}{$<0.001$} \\
\hline Nankana & $392(46.3)$ & $401(40.4)$ & $793(43.1)$ & \\
\hline Minority ethnic groups & $15(1.77)$ & $76(7.65)$ & $91(4.95)$ & \\
\hline \multicolumn{5}{|l|}{ Educational status } \\
\hline No formal education & $517(61.1)$ & $768(77.3)$ & $1285(69.9)$ & \multirow[t]{2}{*}{$<0.001$} \\
\hline Formal education & $329(38.9)$ & $225(22.7)$ & $554(30.1)$ & \\
\hline \multicolumn{5}{|l|}{ Employment status } \\
\hline Unemployed & $292(34.5)$ & $390(39.3)$ & $682(37.1)$ & \multirow{2}{*}{0.035} \\
\hline Employed & $554(65.5)$ & $603(60.7)$ & $1157(62.9)$ & \\
\hline \multicolumn{5}{|l|}{ Marital status } \\
\hline Currently married & $717(84.8)$ & $632(63.7)$ & $1349(73.4)$ & \multirow[t]{2}{*}{$<0.001$} \\
\hline Currently unmarried & $129(15.3)$ & $361(36.4)$ & $490(26.6)$ & \\
\hline \multicolumn{5}{|l|}{ Household SES } \\
\hline Low SES & $625(73.8)$ & $817(82.3)$ & $430(23.4)$ & \multirow[t]{2}{*}{$<0.001$} \\
\hline High SES & $221(26.1)$ & $176(17.7)$ & $397(21.6)$ & \\
\hline Fruit intake (servings/week) & $7(0-7)$ & $7(0-7)$ & $7(0-7)$ & 0.094 \\
\hline Vegetable intake (servings/week) & $21.0(14.0-35.0)$ & $21.0(14.0-28.0)$ & $21.0(14.0-28.0)$ & 0.002 \\
\hline Vendor meals (times/month) & $0.003(0.00-60.0)$ & $0.00(0.00-30.0)$ & $0.00(0.00-60.0)$ & $<0.001$ \\
\hline \multicolumn{5}{|l|}{ Alcohol intake } \\
\hline No alcohol intake & $291(34.4)$ & $606(60.6)$ & $893(48.6)$ & \multirow[b]{2}{*}{$<0.001$} \\
\hline Current alcohol intake & $555(65.6)$ & $391(39.4)$ & $946(51.4)$ & \\
\hline \multicolumn{5}{|l|}{ Tobacco smoking } \\
\hline No smoking & $661(78.1)$ & $979(98.6)$ & $1640(89.2)$ & \multirow[b]{2}{*}{$<0.001$} \\
\hline Current smoking & $185(21.9)$ & $14(1.4)$ & $199(10.9)$ & \\
\hline \multicolumn{5}{|l|}{ Smokeless tobacco } \\
\hline No smokeless tobacco use & $760(89.8)$ & $892(89.8)$ & $1652(89.8)$ & \multirow[b]{2}{*}{0.997} \\
\hline Current smokeless tobacco use & $86(10.2)$ & $101(10.2)$ & $187(10.2)$ & \\
\hline Pesticide exposure & $509(60.2)$ & $497(50.1)$ & $1006(54.7)$ & $<0.001$ \\
\hline \multicolumn{5}{|l|}{ Physical activity } \\
\hline Less physically active & $80(9.46)$ & $182(18.33)$ & $262(14.25)$ & \multirow[t]{2}{*}{$<0.001$} \\
\hline More physically active & $766(90.54)$ & $811(81.67)$ & & \\
\hline MVPA (hours/week) & $41.0(17.0-5.00)$ & $28.0(5.00-44.0)$ & $34.5(9.00-48.0)$ & $<0.001$ \\
\hline Sleeping (hours/week) & $56(49-61)$ & $56(54-63)$ & $56(49-63)$ & $<0.001$ \\
\hline BMI $\left(\mathrm{kg} / \mathrm{m}^{2}\right)$ & $20.5(19.0-22.3)$ & $21.5(19.7-24.0)$ & $20.5(19.0-22.3)$ & $<0.001$ \\
\hline Hip $(\mathrm{cm})$ & $8.30(7.90-8.80)$ & $8.80(8.30-9.40)$ & $8.30(7.90-8.80)$ & $<0.001$ \\
\hline Waist $(\mathrm{cm})$ & $7.20(6.80-7.70)$ & $7.50(7.10-8.20)$ & $7.40(6.90-7.90)$ & $<0.001$ \\
\hline HDL-C (mmol/l) & $1.14(0.90-1.40)$ & $1.08(0.89-1.31)$ & $1.10(0.90-1.35)$ & 0.002 \\
\hline LDL-C (mmol/l) & $1.58(1.12-2.16)$ & $1.61(1.19-2.13)$ & $1.60(1.16-2.14)$ & 0.427 \\
\hline $\mathrm{TC}(\mathrm{mmol} / \mathrm{l})$ & $3.13(2.59-3.70)$ & $3.20(2.69-3.82)$ & $3.16(2.63-3.78)$ & 0.038 \\
\hline TG $(\mathrm{mmol} / \mathrm{l})$ & $0.56(0.43-0.73)$ & $0.55(0.43-0.73)$ & $0.55(0.43-0.73)$ & 0.770 \\
\hline Visceral fat $(\mathrm{cm})$ & $4.03(3.29-4.83)$ & $3.32(2.75-4.10)$ & $3.63(2.99-4.50)$ & $<0.001$ \\
\hline Subcutaneous fat $(\mathrm{cm})$ & $0.70(0.56-0.87)$ & $1.01(0.74-1.45)$ & $0.83(0.62-1.18)$ & $<0.001$ \\
\hline
\end{tabular}

Data shown as $n$ (\%) or median (interquartile range); MVPA: moderate-to-vigorous physical activity.

population. Male gender was associated with higher SBP and PP. Nankana ethnicity was associated with higher PP in men. Women who were currently unmarried had higher levels of all the blood indices compared to married women. Pesticide exposure was associated with reduced levels of all the measured blood pressure indices in women, but not in men. Vendor meal intake in women was associated with higher levels of PP while fruit consumption in men was associated with lower PP levels. Increased abdominal subcutaneous fat was associated with higher DBP and MAP levels in both men and women and with higher SBP levels in men. Increased visceral fat thickness was associated with higher DBP and MAP levels in the total population. In men, increased hip circumference was associated with higher DBP and MAP levels. Increased HDL-C levels were associated with higher SBP, DBP, and MAP in both men and women and higher PP in men.

The increase in blood pressure indices with increasing age in the study population confirms earlier findings in the study area [9], other rural settings in Ghana [34], and elsewhere in sub-Saharan Africa [25, 35, 36]. Increasing levels of blood pressure indices with ageing is attributed to 
TABLE 2: Median levels of blood pressure indices in the rural adult Ghanaian study population stratified by gender and in the combined population.

\begin{tabular}{lcccc}
\hline Variable & Men & Women & Total & $p$ value \\
\hline SBP $(\mathrm{mmHg})$ & $122(111-136)$ & $120(108-135)$ & $121(109-135)$ & 0.007 \\
DBP (mmHg) & $75.5(68.0-84.0)$ & $75.5(68.5-84.5)$ & $75.5(68.0-84.5)$ & 0.719 \\
PP (mmHg) & $46.5(40.5-54.0)$ & $43.5(37.0-52.5)$ & $45.0(38.0-53.0)$ & $<0.001$ \\
MAP (mmHg) & $90.6(82.7-101)$ & $90.0(81.7-101)$ & $90.3(75.7-101)$ & 0.340 \\
\hline
\end{tabular}

Data given as median (interquartile range).

TABLE 3: Multivariable linear regression analysis of factors associated with SBP among men and women and the total population.

\begin{tabular}{lccc}
\hline Variable & Women & Men & Total \\
& $\beta$-coefficient $(95 \% \mathrm{CI})$ & $\beta$-coefficient $(95 \% \mathrm{CI})$ & $\beta$-coefficient $(95 \% \mathrm{CI})$ \\
\hline Age (years) & $0.006(0.004,0.004)^{* * *}$ & $0.005(0.003,0.007)^{* * *}$ & $0.005(0.004,0.007)^{* * *}$ \\
Male & - & - & $0.044(0.026,0.062)^{* * *}$ \\
High SES $^{1 *}$ & - & $-0.007(-0.033,0.018)$ & $-0.005(-0.024,0.014)$ \\
Currently unmarried & $0.038(0.016,0.060)^{* *}$ & - & $0.024(0.007,0.041)^{* *}$ \\
Past or current smoker & - & $-0.019(-0.041,0.003)$ & - \\
Used smokeless tobacco & $0.016(-0.019,0.051)$ & - & $0.015(-0.009,0.040)$ \\
Pesticide exposure & $-0.034(-0.055,-0.013)^{* *}$ & - & $-0.020(-0.036,-0.005)^{* *}$ \\
MVPA (hours/week) & $-0.013(-0.027,0.002)$ & - & $-0.010(-0.021,0.002)$ \\
Fruit (servings/week) & $-0.0002(-0.001,0.001)$ & $-0.0004(-0.001,0.0003)$ \\
BMI (kg/m $\left.{ }^{2}\right)$ & $0.005(-0.001,0.010)$ & $3.96 e-06(-0.004,0.004)$ & $0.002(-0.001,0.006)$ \\
Waist (cm) & $0.015(-0.007,0.036)$ & $-0.002(-0.020,0.016)$ & $0.006(-0.007,0.020)$ \\
Hip (cm) & $-0.005(-0.022,0.012)$ & $0.018(-0.004,0.036)$ & $0.006(-0.007,0.018)$ \\
Visceral fat (cm) & $0.003(-0.008,0.014)$ & $0.008(-0.001,0.017)$ & $0.006(-0.001,0.013)$ \\
Subcutaneous fat $(\mathrm{cm})$ & $0.026(-0.003,0.055)$ & $0.053(0.021,0.086)^{* *}$ & $0.039(0.018,0.060)^{* * *}$ \\
HDL-C (mmol/l) & $0.055(0.021,0.089)^{* *}$ & $0.063(0.034,0.091)^{* * *}$ & $0.054(0.032,0.076)^{* * *}$ \\
LDL-C (mmol/l) & - & $0.001(-0.015,0.017)$ & $-0.011(-0.023,0.001)$ \\
TC (mmol/l) & $-0.003(-0.015,0.010)$ & $0.003(-0.013,0.018)$ & $0.007(-0.004,0.018)$ \\
TG (mmol/l) & $-0.032(-0.064,0.001)$ & $0.013(-0.006,0.031)$ & $0.003(-0.014,0.020)$ \\
\hline
\end{tabular}

All blood pressure components were log-transformed; ${ }^{1}$ SES was coded as those with highest vs. those with lowest SES; ${ }^{2}$ Smoking status was coded as those who are current or past smokers vs. those who never smoked; ${ }^{* * *} p$ value $<0.001 ;{ }^{* *} p$ value $<0.005 ;{ }^{*} p$ value $<0.05$.

TABLE 4: Multivariable linear regression analysis of factors associated with DBP among men and women and the total population.

\begin{tabular}{lccc}
\hline Variable & Women & Men & Total \\
& $\beta$-coefficient $(95 \% \mathrm{CI})$ & $\beta$-coefficient $(95 \% \mathrm{CI})$ & $\beta$-coefficient $(95 \% \mathrm{CI})$ \\
\hline Age (years) & $0.003(0.001,0.005)^{* *}$ & $0.004(0.002,0.006)^{* * *}$ & $0.004(0.002,0.006)^{* * *}$ \\
Nankana ethnicity & $0.001(-0.020,0.021)$ & - & $-0.006(-0.030,0.019)$ \\
Some formal education ${ }^{1}$ & $0.018(-0.005,0.041)$ & - & $0.008(-0.014,0.030)$ \\
Employed & - & $-0.017(-0.040,0.005)$ & - \\
High SES & $-0.010(-0.037,-0.016)$ & $-0.004(-0.030,0.022)$ & $-0.010(-0.036,0.017)$ \\
Currently unmarried & $0.023(0.003,0.043)^{*}$ & - & $-0.001(-0.031,0.029)$ \\
Past or current smoker & $-0.021(-0.074,0.032)$ & - & $-0.009(-0.032,0.014)$ \\
Pesticide exposure & $-0.028(-0.048,-0.009)^{* *}$ & - & $-0.007(-0.031,-0.016)$ \\
MVPA (hours/week) & $-0.008(-0.022,0.006)$ & $-0.019(-0.039,0.002)$ & $-0.018(-0.038,0.003)$ \\
BMI (kg/m $\left.{ }^{2}\right)$ & $0.004(-0.001,0.009)$ & $-0.001(-0.005,0.004)$ & $-0.001(-0.005,0.004)$ \\
Waist $(\mathrm{cm})$ & $0.020(-0.0001,0.040)$ & $0.002(-0.016,0.021)$ & $0.001(-0.018,0.020)$ \\
Hip (cm) & $-0.005(-0.010,0.021)$ & $0.020(0.002,0.039)^{*}$ & $0.020(0.001,0.038)^{*}$ \\
Visceral fat (cm) & $0.003(-0.007,0.013)$ & $0.009(-0.001,0.018)$ & $0.010(0.0001,0.019)^{*}$ \\
Subcutaneous fat $(\mathrm{cm})$ & $0.036(0.009,0.062)^{* *}$ & $0.078(0.045,0.111)^{* * *}$ & $0.076(0.043,0.109)^{* * *}$ \\
HDL-C (mmol/l) & $0.055(0.023,0.086)^{* *}$ & $0.061(0.032,0.091)^{* * *}$ & $0.064(0.033,0.094)^{* * *}$ \\
LDL-C (mmol/l) & $-0.017(-0.033,0.0002)$ & $-0.003(-0.020,0.013)$ & $-0.003(-0.020,0.014)$ \\
TC (mmol/l) & $0.008(-0.007,0.022)$ & $0.003(-0.013,0.019)$ & $0.001(-0.016,0.018)$ \\
TG (mmol/l) & $-0.016(-0.048,0.016)$ & $0.019(-0.001,0.038)$ & $0.019(-0.001,0.038)$ \\
\hline
\end{tabular}

All blood pressure components were log-transformed; ${ }^{1}$ education was coded as some formal education vs. no education; ${ }^{2}$ SES was coded as those with highest vs. those with lowest SES; ${ }^{3}$ smoking status was coded as those who are current or past smokers vs. those who never smoked; ${ }^{* * *} p$ value $<0.001$; ${ }^{* *} p$ value $<0.005 ;{ }^{*} p$ value $<0.05$. 
TABLE 5: Multivariable linear regression analysis of factors associated with MAP among men and women and the total population.

\begin{tabular}{lccc}
\hline Variable & Women & Men & Total \\
& $\beta$-coefficient $(95 \% \mathrm{CI})$ & $\beta$-coefficient $(95 \% \mathrm{CI})$ & $\beta$-coefficient $(95 \% \mathrm{CI})$ \\
\hline Age (years) & $0.004(0.002,0.006)^{* *}$ & $0.004(0.003,0.006)^{* * *}$ & $0.004(0.003,0.005)^{* * *}$ \\
Some formal education $^{1}$ & $0.012(-0.011,0.036)$ & - & - \\
High SES $^{2}$ & $-0.014(-0.041,-0.013)$ & $-0.008(-0.033,0.017)$ & $0.002(-0.016,0.020)$ \\
Currently unmarried $_{\text {Past or current smoker }}^{3}$ & $0.030(0.010,0.051)^{* *}$ & - & $0.011(-0.016,0.020)$ \\
Pesticide exposure & - & $-0.013(-0.035,0.008)$ & - \\
MVPA (hours/week) & $-0.031(-0.050,-0.011)^{* *}$ & - & $-0.016(-0.030,-0.002)^{*}$ \\
BMI (kg/m $\left.{ }^{2}\right)$ & $-0.011(-0.024,0.003)$ & - & $-0.009(-0.020,0.002)$ \\
Waist (cm) & $0.005(-0.001,0.009)$ & $-0.0003(-0.005,0.004)$ & $0.002(-0.002,0.005)$ \\
Hip (cm) & $0.016(-0.004,0.036)$ & $0.0001(-0.018,0.018)$ & $0.008(-0.005,0.022)$ \\
Visceral fat (cm) & $0.001(-0.015,0.016)$ & $0.019(0.001,0.036)^{*}$ & $0.005(-0.007,0.016)$ \\
Subcutaneous fat (cm) & $0.003(-0.008,0.013)$ & $0.009(-0.00003,0.018)$ & $0.012(0.006,0.018)^{* * *}$ \\
HDL-C (mmol/l) & $0.034(0.007,0.060)^{*}$ & $0.066(0.035,0.098)^{* * *}$ & $0.035(0.016,0.054)^{* * *}$ \\
LDL-C (mmol/l) & $0.058(0.026,0.089)^{* * *}$ & $0.062(0.033,0.090)^{* * *}$ & $0.057(0.036,0.078)^{* * *}$ \\
TC (mmol/l) & - & $-0.001(-0.017,0.015)$ & $-0.010(-0.021,0.001)$ \\
TG (mmol/l) & $-0.001(-0.013,0.011)$ & $0.002(-0.013,0.018)$ & $0.006(-0.004,0.016)$ \\
\hline
\end{tabular}

All blood pressure components were log-transformed; ${ }^{1}$ education was coded as some formal education vs. no education; ${ }^{2}$ SES was coded as those with highest vs. those with lowest SES; ${ }^{3}$ smoking status was coded as those who are current or past smokers vs. those who never smoked; ${ }^{* * *} p$ value $<0.001$; ${ }^{* *} p$ value $<0.005 ;{ }^{*} p$ value $<0.05$.

TABLE 6: Multivariable linear regression analysis of factors associated with PP among men and women and the total population.

\begin{tabular}{|c|c|c|c|}
\hline Variable & $\begin{array}{c}\text { Women } \\
\beta \text {-coefficient }(95 \% \mathrm{CI})\end{array}$ & $\begin{array}{c}\text { Men } \\
\beta \text {-coefficient }(95 \% \mathrm{CI})\end{array}$ & $\begin{array}{c}\text { Total } \\
\beta \text {-coefficient }(95 \% \mathrm{CI}) \\
\end{array}$ \\
\hline Age (years) & $0.010(0.007,0.013)^{* *}$ & $0.006(0.003,0.009)^{* * *}$ & $0.009(0.007,0.011)^{* * *}$ \\
\hline Male gender & - & - & $0.079(0.050,0.109)^{* * *}$ \\
\hline Nankana ethnicity & - & $0.037(0.004,0.071)^{*}$ & $0.042(0.018,0.066)^{* *}$ \\
\hline High SES ${ }^{2}$ & $-0.025(-0.070,0.020)$ & - & - \\
\hline Currently unmarried & $0.054(0.020,0.088)^{* *}$ & - & $0.044(0.018,0.070)^{* *}$ \\
\hline Some formal education ${ }^{1}$ & $0.001(-0.038,0.041)$ & - & - \\
\hline Past or current smoker ${ }^{2}$ & - & $-0.010(-0.031,0.011)$ & $-0.010(-0.030,0.011)$ \\
\hline Used smokeless tobacco & $0.023(-0.031,0.077)$ & $0.026(-0.024,0.077)$ & $0.023(-0.014,0.061)$ \\
\hline Pesticide exposure & $-0.042(-0.072,-0.009)^{*}$ & - & $-0.011(-0.035,0.013)$ \\
\hline Vendor (meals/month) & $0.0004(0.00002,0.0008)^{*}$ & - & - \\
\hline Fruit (servings/week) & - & $-0.001(-0.003,-0.00001)^{*}$ & $-0.001(-0.002,0.0003)$ \\
\hline MVPA (hours/week) & $-0.019(-0.042,0.004)$ & $0.018(-0.011,0.047)$ & $-0.009(-0.027,0.009)$ \\
\hline Sleeping (hours/week) & - & $0.001(-0.001,0.003)$ & - \\
\hline $\operatorname{Hip}(\mathrm{cm})$ & $0.005(-0.013,0.022)$ & - & $0.011(-0.002,0.024)$ \\
\hline Visceral fat $(\mathrm{cm})$ & - & $0.011(-0.001,0.024)$ & - \\
\hline HDL-C (mmol/l) & $0.044(-0.003,0.091)$ & $0.044(0.001,0.087)^{*}$ & $0.048(0.014,0.082)^{*}$ \\
\hline LDL-C (mmol/l) & - & $0.005(-0.020,0.029)$ & - \\
\hline $\mathrm{TC}(\mathrm{mmol} / \mathrm{l})$ & - & $0.017(-0.007,0.042)$ & $0.007(-0.007,0.021)$ \\
\hline
\end{tabular}

All blood pressure components were log-transformed; ${ }^{1}$ education was coded as some formal education vs. no education; ${ }^{2}$ smoking status was coded as those who are current or past smokers vs. those who never smoked; ${ }^{* * *} p$ value $<0.001 ;{ }^{* *} p$ value $<0.005 ;{ }^{*} p$ value $<0.05$.

several pathophysiological factors such as decreased baroreceptor sensitivity, increased responsiveness to sympathetic nervous system stimuli, and altered sodium metabolism resulting in increased salt sensitivity [37, 38]. Ageing is associated with changes in large artery stiffness and increased peripheral vascular resistance resulting in increasing SBP and DBP, respectively $[39,40]$.

The higher SBP in men compared to women observed in our study is consistent with earlier results in the study setting [9] and in other African settings [27, 28, 41]. While the higher PP among men in our study resonates with some of these African findings [28] other results showed no gender difference in PP [27]. The higher PP in the Nankana ethnic group suggests an influence of ethnicity on blood pressure levels and this is consistent with findings elsewhere [42, 43]. The ethnic differences in blood pressure may be attributed to the potential influence of genetic factors [42].

Our study has shown that being an unmarried woman was associated with high levels of blood pressure indices. This is similar to findings in African American [44] and Arab communities [45] where single or widowed women had higher blood pressure compared to married women. Being married is reported to be protective against adverse health outcomes [46] including CVDs [46, 47]. The higher levels of 
blood pressure indices among unmarried women may be attributed partly to stress associated with a lack of social support [48].

The reason for the lower levels of all the blood pressure indices with pesticide exposure among women is not known and further studies, involving the type and blood concentrations of pesticides, are required to explain the possible mechanisms underlying this observation. There are mixed findings on blood pressure and pesticide exposure in the literature. Thus, some studies have reported higher blood pressure in response to pesticide exposure $[49,50]$ while others have reported contrary findings [51, 52].

The negative association of PP with fruit consumption among men and the positive association of vendor meal consumption with PP among men in the study population support previous findings [53-55] and confirm that healthier diet choices can reduce the burden of hypertension [56] and reduce CVD risk [57].

In this population subcutaneous fat thickness was associated with higher SBP levels in men while both subcutaneous and visceral fat thickness, in both genders, were associated with higher DBP and MAP levels with subcutaneous fat being the adiposity marker with the strongest association. There are many proposed mechanisms for the association of obesity with hypertension [58] with one of the major hypotheses involving the effect of leptin on sympathetic neural activation [59]. This is relevant to the findings in the current study as subcutaneous adipose tissue has been shown to be the main source of leptin in females [60] and this fat depot is the primary determinant of serum leptin levels [61]. However, leptin was not measured in the present study and therefore we are not able to test the hypothesis that leptin mediates the effect of subcutaneous adipose tissue on blood pressure parameters. The positive association if hip circumference with DBP was unexpected as the hip is known to be protective against CVD risk $[62,63]$. Further studies in this population are therefore required to confirm this.

The increase in blood pressure indices with increasing HDL-C levels in this population is supported by data from Burkina Faso [64]. This raises the question of whether low $\mathrm{HDL}-\mathrm{C}$ is truly a reliable predictor of CVD risk in rural northern Ghana.

The strength of this study is that it is the first to investigate the effect of a wide range of factors on an array of blood pressure indices in a rural African adult population. It therefore provides new knowledge on the factors associated with high blood pressure in black Africans. However the study is not without limitations. Causality cannot be inferred due to the cross-sectional design of the study. Interpretation of the results from the questionnaire data should be taken with caution due to possible recall bias from the self-reported responses of the participants. Also, despite the wide range of factors investigated in our study including vendor meal, fruit, and vegetable consumption data on salt which is a known risk factor of hypertension [65] was not investigated. Another limitation of the study was that data on the composition and extent or duration of exposure of the pesticides were not collected.

\section{Conclusion}

Our study demonstrates that SBP, DBP, MAP, and PP are driven by a number of modifiable risk factors which have important implications for possible public health intervention projects. The positive association of subcutaneous fat with blood pressure indices may be indicative of an emerging problem in this rural population and calls for interventions to curb obesity among the study population. Lifestyle interventions specifically fruit intake which is associated with reduced PP among men should be adopted, and vendor meal consumption which is associated with higher PP among women should be avoided to reduce CVD risk among the study population. The higher BP indices among unmarried women call for the need for further studies to identify appropriate healthcare interventions targeted at widows, single women, and women with less social support in rural communities. The lower blood pressure indices associated with pesticide exposure among women calls for further evaluation of the use of pesticides in the community, since these may pose the risk of hypotension among women. The ethnic difference in blood pressure indices in the study population may suggest the potential influence of genetic factors.

\section{Data Availability}

All data analyzed during this study are included within this published article in the supplementary files.

\section{Conflicts of Interest}

The authors declare that they have no conflicts of interest.

\section{Authors' Contributions}

GA, LA, EN, CD, and AO collected the data. GA analyzed the data and drafted the manuscript. EN, LA, AO, NC, and $\mathrm{MR}$ critically reviewed the analyses. LA, EN, CD, MK, AO, SA, NC, and MR edited the draft. All authors read and approved the manuscript.

\section{Acknowledgments}

The AWI-Gen Collaborative Centre was funded by the National Human Genome Research Institute (NHGRI), the Office of the Director (OD), the Eunice Kennedy Shriver National Institute Of Child Health and Human Development (NICHD), the National Institute of Environmental Health Sciences (NIEHS), the Office of AIDS Research (OAR), and the National Institute of Diabetes and Digestive and Kidney Diseases (NIDDK), of the National Institutes of Health (NIH) under award number U54HG006938 and its supplements, as part of the H3Africa Consortium. Additional funding was leveraged from the Department of Science and Technology, South Africa, award number DST/ CON 0056/2014. The authors express their appreciation to the study participants who provided data and samples to this project and the chiefs and elders of the Kassena-Nankana traditional area whose cooperation and support made this 
study successful. The authors appreciate the staff and management of the Navrongo Health Research Centre (NHRC), Ghana, and the Sydney Brenner Institute for Molecular Bioscience (SBIMB), University of the Witwatersrand, South Africa, who have provided the platform, material, and human support for this project. The authors particularly acknowledge both Eric Fato and Immaculate Anati of the NHRC who played key roles in generating the study data, Felix Kondayire of the NHRC who produced the map of the study area, and Freedom Mokumana of the SBIMB who was instrumental in managing the AWI-Gen project data of which the data for this study are a subset. The authors are grateful to Osman Sankoh formally of the INDEPTH Secretariat in Accra, Ghana, for diverse support towards the AWI-Gen study.

\section{Supplementary Materials}

Table S1: univariable linear regression analysis of factors associated with SBP, DBP, MAP, and PP in the total population. Table S2: univariate linear regression analysis of factors associated with SBP, DBP, MAP, and PP among rural northern Ghanaian women. Table S3: univariate linear regression analysis of factors associated with SBP, DBP, MAP, and PP among rural northern Ghanaian men. (Supplementary Materials)

\section{References}

[1] S. Yusuf, S. Rangarajan, K. Teo et al., "Cardiovascular risk and events in 17 low-, middle-, and high-income countries," New England Journal of Medicine, vol. 371, no. 9, pp. 818-827, 2014.

[2] M. Forouzanfar, H. R. Dajani, V. Z. Groza, M. Bolic, S. Rajan, and I. Batkin, "Oscillometric blood pressure estimation: past, present, and future," IEEE Reviews in Biomedical Engineering, vol. 8, pp. 44-63, 2015.

[3] J. Addo, L. Smeeth, and D. A. Leon, "Hypertension in subsaharan Africa," Hypertension, vol. 50, no. 6, pp. 1012-1018, 2007.

[4] M. J. Bloch, "Worldwide prevalence of hypertension exceeds 1.3 billion," Journal of the American Society of Hypertension, vol. 10, no. 10, pp. 753-754, 2016.

[5] W. K. Bosu, "Epidemic of hypertension in Ghana: a systematic review," BMC Public Health, vol. 10, no. 1, p. 418, 2010.

[6] P. Lamptey, A. Laar, A. J. Adler et al., "Evaluation of a community-based hypertension improvement program (ComHIP ) in Ghana: data from a baseline survey," $B M C$ Public Health, vol. 17, no. 1, pp. 1-16, 2017.

[7] J. Addo, A. G. Amoah, and K. A. Koram, "The changing patterns of hypertension in Ghana: a study of four rural communities in the Ga district," Ethnicity and Disease, vol. 16, no. 4, pp. 894-899, 2006.

[8] P. Lloyd-Sherlock, J. Beard, N. Minicuci, S. Ebrahim, and S. Chatterji, "Hypertension among older adults in low- and middle-income countries: prevalence, awareness and control," International Journal of Epidemiology, vol. 43, no. 1, pp. 116-128, 2014.

[9] S. Kunutsor and J. Powles, "Descriptive epidemiology of blood pressure in a rural adult population in northern Ghana," Rural Remote Health, vol. 9, no. 2, p. 1095, 2009.
[10] F. X. Gómez-Olivé, S. A. Ali, F. Made et al., "Regional and sex differences in the prevalence and awareness of hypertension," Global Heart, vol. 12, no. 2, 2017.

[11] O. A. Sanuade, S. Boatemaa, and M. K. Kushitor, "Hypertension prevalence, awareness, treatment and control in Ghanaian population: evidence from the Ghana demographic and health survey," PLoS One, vol. 13, no. 11, Article ID e0205985, 2018.

[12] G. F. Mitchell, L. A. Moyé, E. Braunwald et al., "Sphygmomanometrically determined pulse pressure is a powerful independent predictor of recurrent events after myocardial infarction in patients with impaired left ventricular function," Circulation, vol. 96, no. 12, pp. 4254-4260, 1997.

[13] S. S. Franklin, S. A. Khan, N. D. Wong, M. G. Larson, and D. Levy, "Is pulse pressure useful in predicting risk for coronary heart disease?" Circulation, vol. 100, no. 4, pp. 354-360, 1999.

[14] H. D. Sesso, M. J. Stampfer, B. Rosner et al., "Systolic and diastolic blood pressure, pulse pressure, and mean arterial pressure as predictors of cardiovascular disease risk in men," Hypertens, vol. 36, no. 5, pp. 801-807, 2000.

[15] A. W. Haider, M. G. Larson, S. S. Franklin, and D. Levy, "Systolic blood pressure, diastolic blood pressure, and pulse pressure as predictors of risk for congestive heart failure in the framingham heart study," Annals of Internal Medicine, vol. 138, no. 1, pp. 10-16, 2003.

[16] E. Meaney, F. Alva, R. Moguel, A. Meaney, J. Alva, and R. Webel, "Formula and nomogram for the sphygmomanometric calculation of the mean arterial pressure," Heart, vol. 84, no. 1, p. 64, 2000.

[17] S. S. Franklin, V. A. Lopez, N. D. Wong et al., "Single versus combined blood pressure components and risk for cardiovascular disease," Circulation, vol. 119, no. 2, pp. 243-250, 2009.

[18] K. Bonaa and D. Thelle, "Association between blood pressure and serum lipids in a population: the tromsø Study," Circulation, vol. 83, no. 4, pp. 1305-1314, 1991.

[19] G. Z. Longo, J. Neves, V. M. Luciano, and M. A. Peres, "Prevalence of high blood pressure levels and associated factors among adults in southern Brazil," Arquivos Brasileiros de Cardiologia, vol. 93, no. 4, pp. 360-366, 2009.

[20] A. Chandra, I. J. Neeland, J. D. Berry et al., "The relationship of body mass and fat distribution with incident hypertension," Journal of the American College of Cardiology, vol. 64, no. 10, pp. 997-1002, 2014.

[21] I. S. Afolabi, S. N. Chinedu, E. E. J. Iweala, O. O. Ogunlana, and D. E. Azuh, "Body mass index and blood pressure in a semi-urban community in Ota , Nigeria," Food and Public Health, vol. 5, no. 5, pp. 157-163, 2015.

[22] R. A. Pobee, W. A. Plahar, and W. B. Owusu, "Association between anthropometry and blood pressure among female teachers of child-bearing age in Ghana," Journal of Biology Agriculture and Healthcare, vol. 3, no. 3, pp. 197-208, 2013.

[23] I. Owusu, C. Aryee, W. Owiredu, J. Osei-Yeboah, E. OwusuDabo, and E. Laing, "Analysis of atherogenic and anthropometric profiles of normotensive and hypertensive Ghanaians in the kumasi metropolis," British Journal of Medicine and Medical Research, vol. 7, no. 5, pp. 378-397, 2015.

[24] B. J. C. Onwubere, E. C. Ejim, C. I. Okafor et al., "Pattern of blood pressure indices among the residents of a rural community in south east Nigeria," International Journal of Hypertension, vol. 2011, Article ID 621074, 6 pages, 2011.

[25] A. O. Sola, O. I. Chinyere, A. O. Stephen, and J. A. Kayode, "Hypertension prevalence in an urban and rural area of 
Nigeria," Journal of Medicine and Medical Sciences, vol. 4, no. 4, pp. 149-154, 2013.

[26] W. K. Bosu, "Determinants of mean blood pressure and hypertension among workers in west Africa," International Journal of Hypertension, vol. 2016, Article ID 3192149, 19 pages, 2016.

[27] P. B. Kinau, M. E. Mpembele, V. E. Kintoki, and I. N. F. M. K.-R. L.-M. B. Mbutiwi, "iMedPub journals correlations between pulse pressure and anthropometric indices of Obesity: cross-sectional study in a congolese southwest port city abstract," Cardiovascular Research, vol. 1, no. 1, p. 2, 2017.

[28] G. S. Bimenya, W. Byarugaba, S. Kalungi et al., "Blood pressure profiles among makerere university undergraduate students," African Health Sciences, vol. 5, no. 2, pp. 99-106, 2005.

[29] A. R. Oduro, G. Wak, D. Azongo et al., "Profile of the navrongo health and demographic surveillance system," International Journal of Epidemiology, vol. 41, no. 4, pp. 968976, 2012

[30] M. Ramsay, N. Crowther, E. Tambo et al., "H3Africa AWIGen collaborative centre: a resource to study the interplay between genomic and environmental risk factors for cardiometabolic diseases in four sub-Saharan African countries," Global Health, Epidemiology and Genomics, vol. 1, p. e20, 2016.

[31] G. Agongo, E. A. Nonterah, C. Debpuur et al., "The burden of dyslipidaemia and factors associated with lipid levels among adults in rural northern Ghana: an AWI-Gen sub-study," PLoS One, vol. 13, no. 11, Article ID e0206326, 2018.

[32] S. A. Ali, C. Soo, G. Agongo et al., "Genomic and environmental risk factors for cardiometabolic diseases in Africa: methods used for phase 1 of the AWI-Gen population crosssectional study," Global Health Action, vol. 11, no. sup2, Article ID 1507133, 2018.

[33] P. A. Harris, R. Taylor, R. Thielke, J. Payne, N. Gonzalez, and J. G. Conde, "Research electronic data capture (REDCap)-a metadata-driven methodology and workflow process for providing translational research informatics support," Journal of Biomedical Informatics, vol. 42, no. 2, pp. 377-381, 2009.

[34] C. Agyemang, W. K. Redekop, E. Owusu-Dabo, and M. A. Bruijnzeels, "Blood pressure patterns in rural, semiurban and urban children in the Ashanti region of Ghana, West Africa," BMC Public Health, vol. 5, no. 1, pp. 1-7, 2005.

[35] S. O. Bushara, S. K. Noor, W. M. Elmadhoun, A. A. Sulaiman, and M. H. Ahmed, "Undiagnosed hypertension in a rural community in Sudan and association with some features of the metabolic syndrome: how serious is the situation?" Renal Failure, vol. 37, no. 6, pp. 1022-1026, 2015.

[36] A. R. Isara and P. O. Okundia, "The burden of hypertension and diabetes mellitus in rural communities in Southern Nigeria," Pan African Medical Journal, vol. 20, p. 103, 2015.

[37] M. A. Weber, J. M. Neutel, and D. G. Cheung, "Hypertension in the aged: a pathophysiologic basis for treatment," The American Journal of Cardiology, vol. 63, no. 16, p. 25, 1989.

[38] M. H. Weinberger and N. S. Fineberg, "Sodium and volume sensitivity of blood pressure. Age and pressure change over time," Hypertension, vol. 18, no. 1, pp. 67-71, 1991.

[39] J. Mayet and A. Hughes, "Cardiac and vascular pathophysiology in hypertension," Heart, vol. 89, no. 9, pp. 1104-1109, 2003.

[40] L. R. Mikael, A. M. G. Paiva, M. M. Gomes et al., "Vascular aging and arterial stiffness," Arquivos Brasileiros de Cardiologia, vol. 109, no. 109, pp. 253-258, 2017.
[41] K. W. Ongeti, J. A. Ogeng, A. N. Pulei, B. O. Olabu, and C. N. Gakara, "Blood pressure characteristics among slum dwellers in Kenya," Global Advanced Research Journal of Microbiology, vol. 2, no. 4, pp. 80-85, 2013.

[42] N. Kato, "Ethnic differences in genetic predisposition to hypertension," Hypertension Research, vol. 35, no. 6, pp. 574-581, 2012.

[43] L. Holmes, J. Hossain, D. Ward, and F. Opara, "Racial/ethnic variability in hypertension prevalence and risk factors in national health interview survey," ISRN Hypertension, vol. 2013, no. 8, Article ID 257842, 2013.

[44] H. M. Schwandt, J. Coresh, and M. J. Hindin, "Marital status, hypertension, coronary heart disease, diabetes, and death among African American women and men: incidence and prevalence in the atherosclerosis risk in communities (ARIC) study participants," Journal of Family Issues, vol. 31, no. 9, pp. 1211-1229, 2016.

[45] K. Abu-Saad, A. Chetrit, S. Eilat-Adar et al., "Blood pressure level and hypertension awareness and control differ by marital status, sex, and ethnicity: a population-based study," American Journal of Hypertension, vol. 27, no. 12, pp. 1511-1520, 2014.

[46] J. K. Kiecolt-Glaser and T. L. Newton, "Marriage and health: his and hers," Psychological Bulletin, vol. 127, no. 4, pp. 472-503, 2001.

[47] L. J. Waite and E. L. Lehrer, "The benefits from marriage and religion in the United States: a comparative analysis," Population and Development Review, vol. 29, no. 2, pp. 255-275, 2003.

[48] F. Ozbay, D. C. Johnson, E. Dimoulas, C. A. Morgan, D. Charney, and S. Southwick, "Social support and resilience to stress: from neurobiology to clinical practice," Psychiatry (Edgmont (Pa.: Township)), vol. 4, no. 5, pp. 35-40, 2007.

[49] A. Goncharov, M. Pavuk, H. R. Foushee, and D. O. Carpenter, "Blood pressure in relation to concentrations of PCB congeners and chlorinated pesticides," Environmental Health Perspectives, vol. 119, no. 3, pp. 319-325, 2011.

[50] N. Samsuddin, K. G. Rampal, N. H. Ismail, N. Z. Abdullah, and H. E. Nasreen, "Pesticide exposure and cardiovascular hemodynamic parameters among male workers involved in mosquito control in east coast of Malaysia," American Journal of Hypertension, vol. 29, no. 2, 2016.

[51] J. Davies, D. Roberts, P. Eyer, N. Buckley, and M. Eddleston, "Hypotension in severe dimethoate self-poisoning," Clinical Toxicology, vol. 46, no. 9, pp. 880-884, 2008.

[52] L. A. Henríquez-Hernández, O. P. Luzardo, M. Zumbado et al., "Blood pressure in relation to contamination by polychlorobiphenyls and organochlorine pesticides: results from a population-based study in the Canary Islands (Spain)," Environmental Research, vol. 135, pp. 48-54, 2014.

[53] S. Kar and B. Khandelwal, "Fast foods and physical inactivity are risk factors for obesity and hypertension among adolescent school children in east district of Sikkim, India," Journal of Natural Science, Biology and Medicine, vol. 6, no. 2, pp. 356-359, 2015.

[54] B. Lea, M. Isao, S. Ambika, W.C. Willett, E. B. Rimm, and J. P. Forman, "Fruit and vegetable consumption and the incidence of hypertension in three prospective cohort studies," Hypertension, vol. 67, no. 2, pp. 288-293, 2016.

[55] C.-N. Zhao, X. Meng, Y. Li et al., "Fruits for prevention and treatment of cardiovascular diseases," Nutrients, vol. 9, no. 6, p. 598, 2017. 
[56] L. A. Bazzano, T. Green, T. N. Harrison, and K. Reynolds, "Dietary approaches to prevent hypertension," Current Hypertension Reports, vol. 15, no. 6, pp. 694-702, 2013.

[57] P. Parikh, M. C. McDaniel, M. D. Ashen et al., "Diets and cardiovascular disease," Journal of the American College of Cardiology, vol. 45, no. 9, pp. 1379-1387, 2005.

[58] V. G. DeMarco, A. R. Aroor, and J. R. Sowers, "The pathophysiology of hypertension in patients with obesity," Nature Reviews Endocrinology, vol. 10, no. 6, pp. 364-376, 2014.

[59] B. B. Bell and K. Rahmouni, "Leptin as a mediator of obesityinduced hypertension," Current Obesity Reports, vol. 5, no. 4, pp. 397-404, 2016.

[60] V. Van Harmelen, S. Reynisdottir, P. Eriksson et al., "Leptin secretion from subcutaneous and visceral adipose tissue in women," Diabetes, vol. 47, no. 6, pp. 913-917, 1998.

[61] A. Minocci, G. Savia, R. Lucantoni et al., "Leptin plasma concentrations are dependent on body fat distribution in obese patients," International Journal of Obesity, vol. 24, no. 9, pp. 1139-1144, 2000.

[62] M. B. Snijder, P. Z. Zimmet, M. Visser, J. M. Dekker, J. C. Seidell, and J. E. Shaw, "Independent and opposite associations of waist and hip circumferences with diabetes, hypertension and dyslipidemia: the AusDiab Study," International Journal of Obesity, vol. 28, no. 3, pp. 402-409, 2004.

[63] J. B. Ruige and L. F. Van Gaal, "Low fasting triglycerides: hallmark of the healthy large hip?" Obesity, vol. 17, no. 8, pp. 1621-1626, 2009.

[64] A. T. C. R. K. Koumaré, A. Guira, A. Samandoulougou et al., "Plasma lipid profile including the high density lipoprotein ( HDL ) subclasses in hypertensive patients in Ouagadougou," Burkina Faso. African Journal of Biochemistry Research, vol. 9, no. 3, pp. 47-54, 2015.

[65] J. Arcand, M. M. Y. Wong, J. A. Santos et al., "More evidence that salt increases blood pressure and risk of kidney disease from the science of salt: a regularly updated systematic review of salt and health outcomes (april-july 2016)," The Journal of Clinical Hypertension, vol. 19, no. 8, pp. 813-823, 2017. 